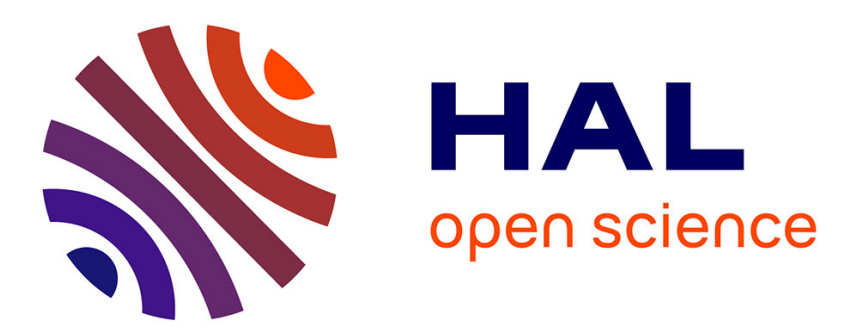

\title{
Migrating Epithelial Monolayer Flows Like a Maxwell Viscoelastic Liquid
}

S. Tlili, M. Durande, Cyprien Gay, B. Ladoux, F. Graner, H. Delanoe-Ayari

\section{To cite this version:}

S. Tlili, M. Durande, Cyprien Gay, B. Ladoux, F. Graner, et al.. Migrating Epithelial Monolayer Flows Like a Maxwell Viscoelastic Liquid. Physical Review Letters, 2020, 125 (8), pp.088102. 10.1103/PhysRevLett.125.088102 . hal-02928890

\section{HAL Id: hal-02928890 https://hal.science/hal-02928890}

Submitted on 11 Feb 2021

HAL is a multi-disciplinary open access archive for the deposit and dissemination of scientific research documents, whether they are published or not. The documents may come from teaching and research institutions in France or abroad, or from public or private research centers.
L'archive ouverte pluridisciplinaire HAL, est destinée au dépôt et à la diffusion de documents scientifiques de niveau recherche, publiés ou non, émanant des établissements d'enseignement et de recherche français ou étrangers, des laboratoires publics ou privés. 


\title{
Migrating Epithelial Monolayer Flows Like a Maxwell Viscoelastic Liquid
}

\author{
S. Tlili, ${ }^{1,2,{ }^{*}}$ M. Durande, ${ }^{1}$ C. Gay,${ }^{1}$ B. Ladoux,${ }^{2,3}$ F. Graner,${ }^{1}$ and H. Delanoë-Ayari $\odot^{4, \dagger}$ \\ ${ }^{1}$ Laboratoire Matière et Systèmes Complexes, Université de Paris-Diderot, \\ CNRS UMR 7057, 10 rue Alice Domon et Léonie Duquet, F-75205 Paris Cedex 13, France \\ ${ }^{2}$ Mechanobiology Institute, Department of Biological Sciences, National University of Singapore, \\ 5A Engineering Drive, 1, 117411 Singapore \\ ${ }^{3}$ Institut Jacques Monod, Université de Paris-Diderot, CNRS UMR 7592, 15 rue Hélène Brion, 75205 Paris Cedex 13, France \\ ${ }^{4}$ Univ. Lyon, Université Claude Bernard Lyon 1, CNRS UMR 5306, Institut Lumière Matière, \\ Campus LyonTech-La Doua, Kastler building, 10 rue Ada Byron, F-69622 Villeurbanne Cedex, France
}

(Received 27 April 2020; accepted 16 July 2020; published 18 August 2020)

\begin{abstract}
We perform a bidimensional Stokes experiment in an active cellular material: an autonomously migrating monolayer of Madin-Darby canine kidney epithelial cells flows around a circular obstacle within a long and narrow channel, involving an interplay between cell shape changes and neighbor rearrangements. Based on image analysis of tissue flow and coarse-grained cell anisotropy, we determine the tissue strain rate, cell deformation, and rearrangement rate fields, which are spatially heterogeneous. We find that the cell deformation and rearrangement rate fields correlate strongly, which is compatible with a Maxwell viscoelastic liquid behavior (and not with a Kelvin-Voigt viscoelastic solid behavior). The value of the associated relaxation time is measured as $\tau=70 \pm 15 \mathrm{~min}$, is observed to be independent of obstacle size and division rate, and is increased by inhibiting myosin activity. In this experiment, the monolayer behaves as a flowing material with a Weissenberg number close to one which shows that both elastic and viscous effects can have comparable contributions in the process of collective cell migration.
\end{abstract}

DOI: 10.1103/PhysRevLett.125.088102

Epithelial tissues are active cellular materials made of constitutive objects, the cells, that can not only deform and exchange neighbors, but also grow, divide, have a polarity, and exert active stresses [1-4]. Tissue mechanical properties have a crucial importance in biological morphogenetic processes [5]. In particular, cell neighbor rearrangements contribute to shaping tissues, as during the Drosophila embryo germ band extension [6]. Rearrangements are often described as either passive, caused by an external stress emerging at the tissue scale, or active, for instance, triggered and directed by an anisotropic distribution of molecules (such as myosin or cadherins) at the cell-cell contacts [7]. Active cell contour fluctuations are key ingredients to fluidize tissues so that they flow under tissuescale stress, both in vitro [8] and in vivo [9].

Experiments performed on embryonic tissues [10], multicellular spheroids $[8,11]$, or cell monolayers with or without substrate $[12,13]$ have suggested describing tissues as viscoelastic liquids. However, there is still a debate around the microscopic origin and value of the viscoelastic relaxation time $\tau$ and on whether Madin-Darby canine kidney (MDCK) monolayers behave predominantly as liquids or solids [12,14]. MDCK cells can actively migrate on a substrate while sustaining tissue cohesivity [13,15-18]. Collective cell migration is due to the cells displaying simultaneously cryptic lamellipodia on their basal side and adherent junctions on their apical side
$[19,20]$. Studying their bidimensional flow in long and narrow adhesive strips facilitates experiments, simulations, theory, and their mutual comparisons [21].

The flow of a liquid relative to a circular obstacle, whether in two or three dimensions, is a classical experiment (introduced by Stokes [22]) to measure a viscosity and/or to probe the mechanical behavior of viscoelastic or viscoplastic materials [23]. More complex materials which are together viscous, elastic, and plastic such as liquid foams (bubbles are able to sustain elastic deformations due to their surface tension, until a yield point where they rearrange and exchange neighbors $[24,25]$ ) have been well characterized using the Stokes experiment, in which upstream bubbles are elongated tangentially to the obstacle and downstream bubbles are elongated radially [26]. The Stokes flow geometry has even been applied in contexts as different as granular materials [27], or active materials, to study clogging in animal crowds [28], or cell-substrate interaction in MDCK cell monolayers, where average traction forces were found to pull the monolayer toward the obstacle [29].

The total deformation rate is the strain rate, also called velocity gradient $\dot{\varepsilon}_{\text {tot }}$. It is the sum of the coarse-grained cell deformation rate and of the intercellular topological change rate (hereafter "rearrangement rate" for short): $\dot{\varepsilon}_{\text {tot }}=\dot{\varepsilon}_{\text {cell }}+$ $\dot{\varepsilon}_{r}$ [30]. The former is the time derivative of the coarsegrained cell deformation field $\varepsilon_{\text {cell }}$, while in the absence of 

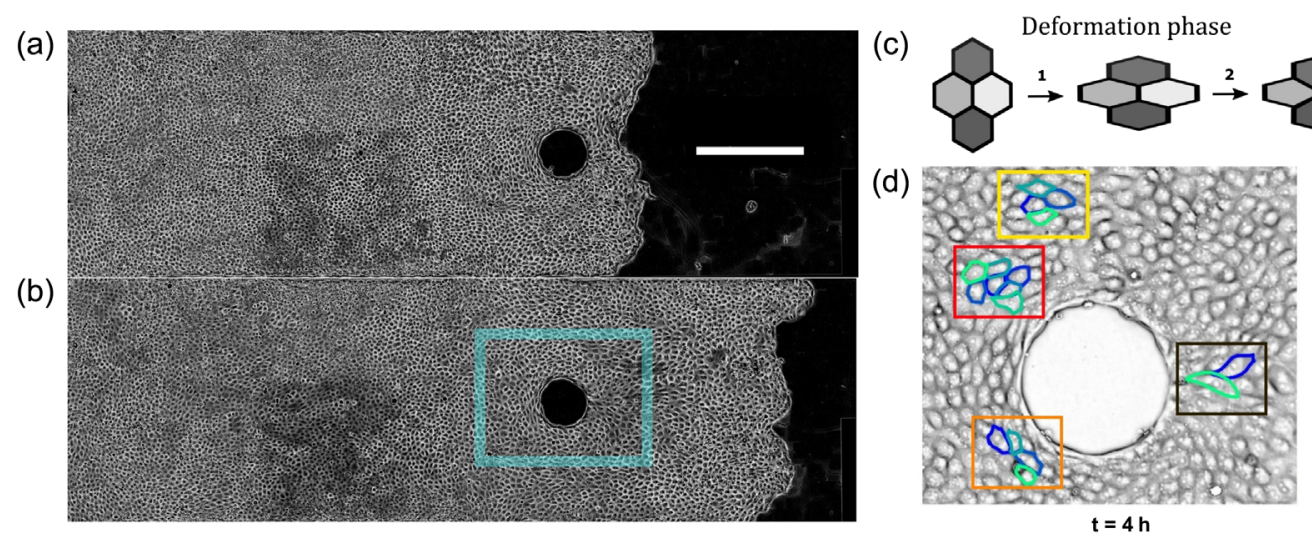

Relaxation phase

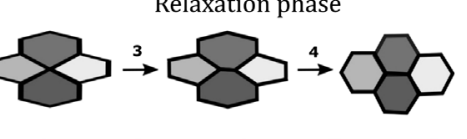

FIG. 1. Stokes flow of migrating cells. Initial time $t=0$ (a), and $t=12 \mathrm{~h}$ later (b), phase contrast images of a monolayer migrating from left to right around a $200 \mu \mathrm{m}$ diameter obstacle (see Movie 1 in Supplemental Material [32]); strip width $1000 \mu \mathrm{m}$, length $4 \mathrm{~mm}$ (the right-hand part is longer than visible on the pictures), scale bar $500 \mu \mathrm{m}$. (c) Sketch of cell deformation and cell-cell rearrangement driven by tissue velocity gradient: (1) cells deform and a cell-cell junction shortens; (2) the cell-cell junction shrinks, and a fourfold vertex is formed; (3) a new pair of neighbor cells is formed (rearrangement); (4) its junction lengthens and cells deform again, during their shape relaxation. (d) Four zones, highlighted by color frames (left, $t=4 \mathrm{~h}$ ), are tracked on $t=0,4,8$, and $12 \mathrm{~h}$ (right), with the same color code, to evidence a few examples of cell-cell rearrangements.

cell divisions and apoptoses, the latter reduces to the deformation rate due to cell-cell rearrangements $\dot{\varepsilon}_{r}$. The Stokes flow, in which these fields are heterogeneous, displays simultaneously a variety of combinations of these fields, depending on the position with respect to the obstacle; for instance, there are regions where the strain rate and deformation are parallel, other regions where they are orthogonal. In that respect, to discriminate between different models, the Stokes flow geometry is more efficient [26] than a homogeneous flow such as a pure shear between parallel boundaries (Couette flow) [31].

Here, we observe over up to one day the Stokes flow of MDCK cell monolayers [Figs. 1(a) and 1(b)] with many cell rearrangements [Figs. 1(c) and 1(d)], and with only few cell divisions thanks to a drug. Direct image analysis methods yield velocity, velocity gradient, and coarse-grained cell anisotropy fields within the frame of continuum mechanics
(Fig. 2). We deduce the rearrangement rate field and quantify the field correlations to probe the tissue rheology (Fig. 3).

The procedure for micropattern printing, cell culture, imaging, and velocity measurement is described in detail in Ref. [14]. Briefly, a strip is $4 \mathrm{~mm}$ long, and it is adhesive, while its four boundaries and the circular obstacle are not. To check reproducibility, each experimental batch is composed of two identical strips. To test the effect of experiment dimensions, three batches are used: the first one with obstacle diameter $150 \mu \mathrm{m}$ and strip width $750 \mu \mathrm{m}$; the second one with obstacle diameter $200 \mu \mathrm{m}$ and strip width $1000 \mu \mathrm{m}$; the third one with obstacle diameter $300 \mu \mathrm{m}$ and strip width $1000 \mu \mathrm{m}$.

We inhibit cell divisions using mitomycin; this prevents cell density increase and jamming that slows down migration. Two hours after having added the mitomycin, we take the first image of the movie and define it as $t=0$. MDCK (a)

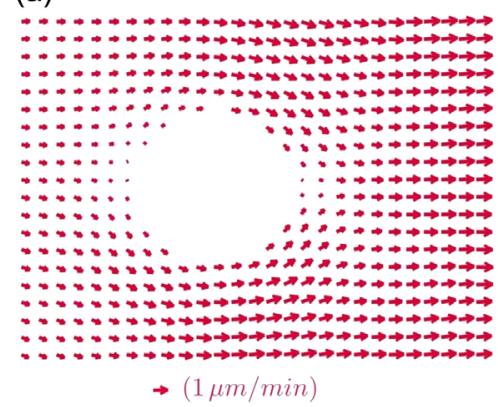

(b)

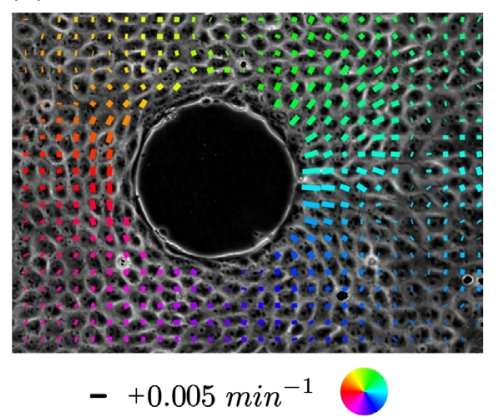

(c)

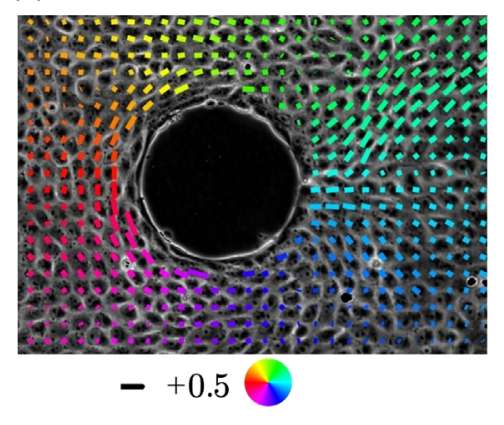

FIG. 2. Cell velocity and deformation field maps around the obstacle. (a) Velocity field $\vec{v}(x, y)$ averaged over $8 \mathrm{~h}$. (b) Deviator of the velocity gradient tensor symmetric part $\nabla v_{\mathrm{sym}}^{\mathrm{dev}}$ obtained by spatial derivation of the velocity field in (a). It is diagonalized and each bar represents its main axis of extension. (c) Deformation tensor deviator $\varepsilon_{\mathbf{e}}^{\text {dev }}$. It is diagonalized and each bar represents its main axis of extension. Same experiment as in Fig. 1, obstacle diameter $200 \mu \mathrm{m}$; scales are indicated below each panel. In (b) and (c) the color codes for the angular position of each point, in polar coordinates originating at the obstacle center. 
(a)

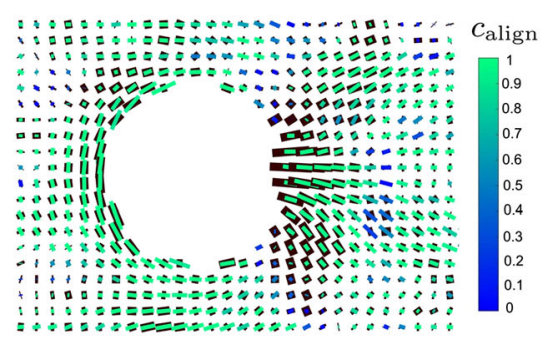

(b)

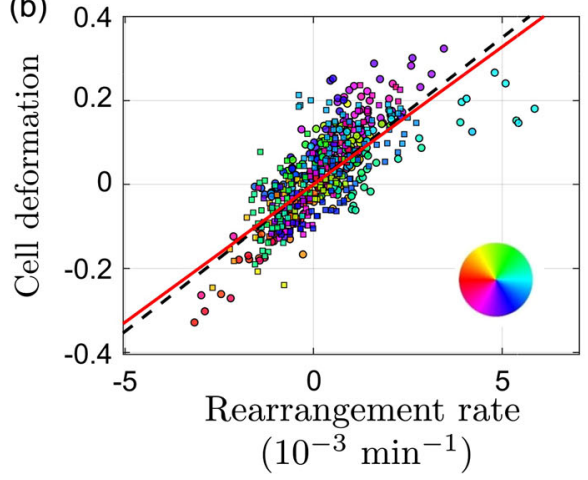

(c)

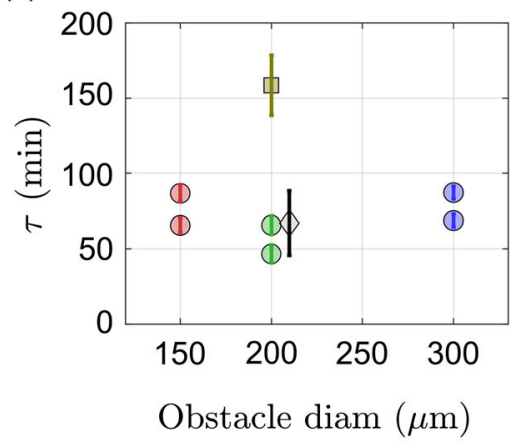

FIG. 3. Test of Maxwell model and measurement of viscoelastic time. (a) Cell deformation versus rearrangement rate: correlation map corresponding to the same individual experiment as in Figs. 1 and 2; obstacle diameter $200 \mu \mathrm{m}$. In black, deviatoric part of the rearrangement rate $\dot{\varepsilon}_{r}$, multiplied by $\tau=70 \mathrm{~min}$. In color, deviatoric part of the cell deformation $\varepsilon_{e}$. The color codes are for the alignment coefficient $c_{\text {align }}$ of these tensors, namely the square cosine of their relative angle, with green corresponding to tensors being aligned $\left(c_{\text {align }}=1\right)$ and blue to tensors being orthogonal $\left(c_{\text {align }}=0\right)$. (b) Cell deformation versus rearrangement rate: components of the tensor deviatoric parts. Circles: $\left\langle\left(\varepsilon_{e}\right)_{x x}-\left(\varepsilon_{e}\right)_{y y}\right\rangle / 2$ plotted versus $\left\langle\left(\dot{\varepsilon}_{r}\right)_{x x}-\left(\dot{\varepsilon}_{r}\right)_{y y}\right\rangle / 2$. Squares: $\left\langle\left(\varepsilon_{e}\right)_{x y}\right\rangle$ plotted versus $\left\langle\left(\dot{\varepsilon}_{r}\right)_{x y}\right\rangle$. Same experiment as in (a), color coding for the box position angle as in Figs. 2(b) and 2(c). Solid red line: linear fit to the data, passing through the origin, slope $68 \mathrm{~min}$; dashed black line: slope $70 \mathrm{~min}$. (c) Circles: values of $\tau$ found for the six strips (Fig. S3 [32]): namely two $150 \mu \mathrm{m}$ obstacles (red), two $200 \mu \mathrm{m}$ obstacles (green), and two $300 \mu \mathrm{m}$ obstacles (blue). Square: with blebbistatin [Figs. S8(a) and S8 (b) [32] ]. Diamond: without mitomycin [Figs. S8(c) and S8(d) [32] ], slightly shifted to the right for legibility.

monolayers can then migrate over millimeters and during many hours at constant total cell number. The 2-mm-long region upstream of the obstacle serves as a cell reservoir; its two-dimensional density is initially high, then decreases. The typical time for cells to migrate over a distance equivalent to the obstacle diameter of $200 \mu \mathrm{m}$ is $3 \mathrm{~h}$. For obstacle diameters of $400 \mu \mathrm{m}$ or larger, the transit time of a cell to migrate across the whole zone influenced by the obstacle is comparable to the experiment duration, so that the steady flow is not completely established. For obstacle diameters of $100 \mu \mathrm{m}$ or smaller, cells often form suspended bridges over the nonadhesive obstacle.

To measure the velocity field and total deformation rate, we divide the field of view in square boxes of 128 pixels per side $(83.2 \mu \mathrm{m})$, labeled by their position $(x, y)$, containing typically 10 cells. We measure the two-dimensional velocity field $\vec{v}(x, y, t)$ using a custom-made MATLAB optic flow code based on the Kanade-Lucas-Tomasi algorithm [33] with a level 2 pyramid. This coarse-grained velocity field can be averaged in time [see Fig. 2(a)], yielding components $v_{i}\left(x_{j}\right)$ where $i, j=1$ or 2 . Using finite differences we obtain in each box the symmetric part of the velocity gradient, $\dot{\varepsilon}_{\text {tot }}=\nabla \vec{v}_{\text {sym }}$, with components $\left(\partial_{i} v_{j}+\partial_{j} v_{i}\right) / 2$. This symmetric tensor can be diagonalized. Its anisotropic part is its deviator $\dot{\varepsilon}_{\text {tot }}^{\text {dev }}=\dot{\varepsilon}_{\text {tot }}-\operatorname{Tr}\left(\dot{\varepsilon}_{\text {tot }}\right) I / 2$, where $I$ is the unit tensor in two dimensions; $\dot{\varepsilon}_{\mathrm{tot}}^{\mathrm{dev}}$ has two opposite eigenvalues. We graphically represent in each box [Fig. 2(b)] this anisotropic part, with bars oriented along the direction of the eigenvector corresponding to the positive eigenvalue (local tissue expansion direction) and of length proportional to this eigenvalue.

To extract the coarse-grained cell anisotropy (i.e., anisotropy of the average cell shape, not to be confused with the average of the cell shape anisotropy) we use Fourier transform (FT) (see Fig. S1 in Supplemental Material [32]); details and validations are provided in Ref. [34]. This method provides an efficient estimation of the coarse-grained elastic deformation field anisotropy without having to recognize and segment each individual cell contour (as in Refs. [35,36]), which is challenging on phase contrast images. We use the same grid and box size as for the velocity. In each box, we multiply the image by a windowing function to avoid singularities in the FT due to boundaries. We then compute the FT using the fast Fourier transform algorithm implemented in MATLAB (fft2.m) and keep only its amplitude (not the phase), which can be time averaged to increase signal-to-noise ratio. We binarize the resulting Fourier space pattern keeping 5\% of the brightest pixels (for justification of this percentile value, see Ref. [34]). We compute the inertia matrix of the binarized FT pattern and diagonalize it, which yields two eigenvalues $\lambda_{\max }^{2}$ and $\lambda_{\min }^{2}$ in the directions of the pattern main axes. They determine the ellipse back in the real space, with eigenvalues $L_{\max }=\left(2 m / \lambda_{\min }\right)$ and $L_{\min }=\left(2 m / \lambda_{\max }\right)$ in the directions of the same axes with $m$ the size of the FT image in pixels.

We define as follows the coarse-grained elastic deformation tensor $\varepsilon_{\mathbf{e}}$ with respect to a rest state which we assume to be isotropic, with two equal eigenvalues $L_{0}=\sqrt{L_{\max } L_{\min }}: \varepsilon_{\mathbf{e}}$ has the same eigenvectors as the Fourier pattern, and two eigenvalues $\frac{1}{2}\left[\left(L_{\max }^{2} / L_{0}^{2}\right)-1\right]$ and $\frac{1}{2}\left[\left(L_{\min }^{2} / L_{0}^{2}\right)-1\right]$. The deformation tensor $\varepsilon_{\mathbf{e}}$ is equivalent to other definitions of the strain (e.g., true strain $[34,37])$ within a linear approximation; in addition, $\varepsilon_{\mathbf{e}}$ has the advantage to have well-established transport equations 
[38]. We represent the anisotropic part of $\varepsilon_{\mathbf{e}}$, namely its deviator $\varepsilon_{\mathbf{e}}^{\mathrm{dev}}$, which only depends on the ratio $\left(L_{\max } / L_{\min }\right)$. This represents the coarse-grained elastic deformation field anisotropy depicted as a bar in Fig. 2(c). In summary, $\varepsilon_{e}^{\text {dev }}$ can easily be measured, and it correctly approximates $\varepsilon_{\mathrm{cell}}^{\mathrm{dev}}$.

Since divisions are inhibited, $\operatorname{Tr}\left(\dot{\varepsilon}_{r}\right)$ is negligible compared with $\dot{\varepsilon}_{r}^{\text {dev }}$ [35]; we have $\operatorname{Tr}\left(\nabla \vec{v}_{\text {sym }}\right) \approx \operatorname{Tr}\left(\dot{\varepsilon}_{\text {cell }}\right)$ and $\nabla \vec{v}_{\mathrm{sym}}^{\mathrm{dev}}=\dot{\varepsilon}_{\mathrm{cell}}^{\mathrm{dev}}+\dot{\varepsilon}_{r}^{\mathrm{dev}}$. Since $\varepsilon_{\mathrm{cell}}^{\mathrm{dev}} \approx \varepsilon_{e}^{\mathrm{dev}}$, we deduce the time averaged rearrangement rate $\left\langle\dot{\varepsilon}_{r}^{\mathrm{dev}}\right\rangle$ by measuring the difference $\left\langle\nabla \vec{v}_{\mathrm{sym}}^{\mathrm{dev}}-\dot{\varepsilon}_{e}^{\mathrm{dev}}\right\rangle$ as in Ref. [30]. From the measurement of $\varepsilon_{e}$, we estimate $\dot{\varepsilon}_{e}$ by taking into account the cell deformation advection in the flow, as $\dot{\varepsilon}_{e}=\frac{D \varepsilon_{e}}{D t}=$ $\frac{\partial \varepsilon_{e}}{\partial t}+\vec{v} \cdot \nabla \varepsilon_{e}$ : this significantly improves the results presented below (we still neglect rotation terms [37,38], which do not change the present results; see Supplemental Material and Fig. S2 [32]). We correlate the deviator of the cell deformation $\left\langle\varepsilon_{e}^{\mathrm{dev}}\right\rangle$ with the deviator of the rearrangement rate $\left\langle\dot{\varepsilon}_{r}{ }^{\text {dev }}\right\rangle$, where both fields are time averaged over the experiment duration (at least $10 \mathrm{~h}$ ).

Qualitatively, to model the flow, we note that cell deformation and rearrangement rate fields correlate strongly [Figs. 3(a) and 3(b)]. This is compatible with a simple Maxwell viscoelastic liquid model where an elastic intracellular component is in series with a viscous intercellular component representing cell rearrangements [38], with the same stress in both elements: $E \varepsilon_{e}=\eta_{r} \dot{\varepsilon}_{r}^{\mathrm{dev}}$. Here $E$ is an effective intracellular Young modulus and $\eta_{r}$ an effective intercellular viscosity due to rearrangements, or equivalently, $\varepsilon_{e}^{\mathrm{dev}}=\dot{\varepsilon}_{r}^{\mathrm{dev}} \tau$ with $\tau=\eta_{r} / E$.

To test the Maxwell model, we compare the anisotropic parts of cell deformation and rearrangement rate fields. Their orientations are identical, except in a region where both fields are small [right of the obstacle on Fig. 3(a), coded in blue]. Their amplitudes are proportional to each other [Figs. 3(a) and 3(b) herein and Fig. S3 in Ref. [32] ]. Their proportionality coefficient is found by linear fit to the data performed with MATLAB robustfit.m. Each individual experiment provides a self-sufficient dataset with a large enough variation range thanks to the Stokes flow heterogeneity. Data with $\varepsilon_{e}^{\text {dev }}$ amplitude smaller than 0.05 correspond to vast, quiet regions of the flow and are excluded from the fit to increase the signal-to-noise ratio. Points at different distances from the obstacle have similar contributions to the measurement of the viscoelastic time (Fig. S4 [32]).

Quantitatively, we find the value $\tau=70 \pm 15 \mathrm{~min}$ [Fig. 3(a)], whichever the fit method: either by fitting the superimposition of all six experiments (Fig. S3 [32], correlation value $R=0.67$ ) or by averaging six experimental fits [Fig. 3(c) herein and Fig. S3 [32], correlations values range from $R=0.53$ to 0.77 ). As expected for a characteristic of the material itself, $\tau$ is independent of the obstacle dimension [Fig. 3(c)]. We also plot $\tau$ versus the monolayer average migration velocity around the obstacle and find that it decreases from around 90 min to below $60 \mathrm{~min}$ (Fig. S5 [32]). If this average velocity is used as a proxy of the deviatoric deformation rate, this suggests that the monolayer rheofluidifies.

To perform an independent and more visual determination of $\tau$, a simulation with a Maxwell model (Fig. S6 [32]) qualitatively reproduces the cell deformations around the obstacle if we use the measured value $\tau=70 \mathrm{~min}$, while taking a much shorter value $\tau=10$ min leads to almost no cell deformation around the obstacle and taking a much longer value $\tau=700$ min leads to cell deformations much higher than the one experimentally observed. A fortiori, our data rule out viscoelastic solid behavior models in which the elastic deformation could be sustained indefinitely (which is the limit of an infinite $\tau$ ). As a third independent test, Fig. S7 [32] rejects the predictions of the Kelvin-Voigt viscoelastic solid model, according to which the time averaged cellular strain rate and the tissue strain rate are equal; if this model was applicable, all data points would collapse on the first bisectrix (black line).

This estimation of $\tau$ could be compared to similar or related measurements from the literature, which for different cell types vary over orders of magnitude [12], and specifically for MDCK monolayers range from $15 \mathrm{~min}$ [39] to $3-5 \mathrm{~h}$ [12]. Our value falls within the range of one to a few hours required to explain the onset of velocity waves and strain waves [14]. The relaxation time $\tau$ we find is associated with cell rearrangements which fluidify the tissue by relaxing cell shapes $[8,38]$.

Note that $\tau$ is much longer than the viscoelastic time associated with intracellular stress dissipation due to cytoskeleton viscosity $(\approx 15 \min [40])$ or cell shape relaxation time (of order of a minute in zebrafish tailbud [10] and even of a second in a suspended MDCK monolayer [13]). A tissue deformation is first transmitted to cell scale within an intracellular timescale, then rearrangements occur at intercellular timescale $[8,41]$. It is the latter time that is probed within the present setup and found to be on the hour timescale.

On the other hand, $\tau$ is much shorter than the viscoelastic time associated with cell division, typically several hours [42]. This suggests that the cell division rate does not play a significant role in the monolayer fluidity, and changing it should not affect $\tau$. Such prediction is successfully tested in Fig. 3(c) herein and Fig. S8 [32], where $\tau$ is insensitive to the mitomycin. In addition, $\tau$ is increased by myosin inhibitor blebbistatin, suggesting that myosin activity contributes to fluidifying the tissue.

To interpret the influence of $\tau$ on the flow, it can be compared to the amplitude of the total deformation rate. The Weissenberg number $\mathrm{We}=\tau\left|\dot{\varepsilon}_{\text {tot }}\right|$ is a dimensionless number characterizing how elasticity affects the flow and how reciprocally the flow affects cell shapes. In tissue regions where $\mathrm{We} \ll 1$ the flow is quasistatic, cell shapes relax and remain close to their rest shape. Conversely, 
wherever We is comparable to or larger than 1, cell shapes depend on the tissue flow history. We find that We reaches its largest value $\approx 0.5$ just downstream of the obstacle (Fig. S9 [32]). This value is at the crossover between these regimes. Elastic and viscous contributions to the flow are comparable. Hence, by regulating the migration velocity and/or the rearrangement dynamics, cells can biologically tune We and the proportion of the elastic deformation they relax. This could be a way to switch progressively from a developing, fluid tissue to a mature, solid tissue [9]. Encoding in the cell shape the memory of the global tissue flow is a way to transmit information from tissue scale to cell scale, and can in turn influence intracellular signaling [43].

The cell shape memory of the global tissue flow appears visually as the extension of the cell deformation wake, far downstream of the obstacle [see Fig. 2(c)], compared with the deformation rate causing this cell deformation which is much more localized near the obstacle [Fig. 2(b)]. Cell deformation is advected downstream at a velocity of order of $1 \mu \mathrm{m} / \mathrm{min}$ [Fig. 2(a)] and its principal source of decay is rearrangements since the total deformation rate essentially vanishes in this region. This competition between deformation advection and rearrangements defines a characteristic length scale $\tau v$. This length scale is $\approx 100 \mu \mathrm{m}$; i.e., it has the same order of magnitude as the correlation length scale usually observed in a migrating MDCK cell monolayer [21].

A tissue which solidifies during its maturation acquires a yield strain, validating the foam-tissue analogy [9]. However, here, although the flow is visually similar to that of a soap froth [26], we do not find such a yield strain. Indeed, the graph of $\varepsilon_{e}$ versus $\dot{\varepsilon}_{r}$ does not display any threshold behavior. In a developing tissue or in collective cell migration, neighbor changes do occur even at low applied deformation, and fluidify the material. The velocity field results from cell activity, more precisely the interplay between collective migration in a band and boundary conditions imposed by the obstacle (as opposed to the one-dimensional migration velocity in an obstacle free band, which only depends on local cell density [14]).

The Stokes flow geometry offers several advantages. It enables us to establish a stationary heterogeneous flow pattern, induce rearrangements, probe the monolayer rheology, discriminate between different models, and measure the viscoelastic time. Our analysis method is based on the flow spatial heterogeneity, with a high enough Weissenberg number, to observe cell deformation, deformation rate, and rearrangement fields with a variety of amplitudes and orientations, in several boxes. Flow stability in time (obtained here thanks to mitomycin) enables data averaging over a few successive images to improve the signal-to-noise ratio. Provided these requirements are met, our analysis is likely easier to apply to monolayers than other existing techniques to measure $\tau$ such as injected functionalized droplets $[9,10]$ or tissue compression $[44,45]$. Measured fields are spatially smooth, their signal-to-noise ratio sufficient to calculate time and space derivatives. Despite the actual velocity fluctuations, it is possible to compute the transport terms of elastic deformation and integrate them along velocity field lines. This enables us to determine all fields involved in a continuum mechanics description, without requiring any theoretical a priori assumption on the velocity fields.

To extend this method in vivo, an experimental technique to introduce an obstacle in a Drosophila embryo has been recently developed: laser-induced tissue cauterization burns a group of cells, attaching it to the vitelline membrane surrounding the embryo [46]. Such mechanical perturbation can help unveil the underlying cause of the morphogenetic tissue flow. In the same spirit, magnetic fluid drops could be introduced as obstacles in 3D tissues such as in zebrafish embryo $[9,10]$. Finally, the present analysis method could be used to analyze a natural motion where a tissue flows around a small organ embedded in it [47].

We thank Sri Ram Krishna Vedula for preliminary experiments that inspired this work, Ibrahim Cheddadi, Philippe Marcq, and Pierre Saramito for stimulating discussions. B. L. acknowledges financial support from the European Research Council under the European Union's Seventh Framework Programme (FP7/2007-2013)/ERC Grant Agreement No. 617233 and Agence Nationale de la Recherche (ANR) "POLCAM" (ANR-17-CE13-0013).

* Present address: Institut de Biologie du Développement de Marseille, UMR 7288, Case 907, Parc Scientifique de Luminy, 13288 Marseille Cedex 9, France. sham.tlili@univ-amu.fr helene.delanoe-ayari@univ-lyon1.fr

[1] S. M. Zehnder, M. Suaris, M. M. Bellaire, and T.E. Angelini, Biophys. J. 108, 247 (2015).

[2] A. Puliafito, L. Hufnagel, P. Neveu, S. Streichan, A. Sigal, D. K. Fygenson, and B. I. Shraiman, Proc. Natl. Acad. Sci. U.S.A. 109, 739 (2012).

[3] B. Ladoux, R.-M. Mège, and X. Trepat, Trends Cell Biol. 26, 420 (2016).

[4] A. Doostmohammadi, S. P. Thampi, T. B. Saw, C. T. Lim, B. Ladoux, and J. M. Yeomans, Soft Matter 11, 7328 (2015).

[5] C.-P. Heisenberg and Y. Bellaïche, Cell 153, 948 (2013).

[6] C. Collinet, M. Rauzi, P. Lenne, and T. Lecuit, Nat. Cell Biol. 17, 1247 (2015).

[7] B. Guirao and Y. Bellaïche, Curr. Opin. Cell Biol. 48, 113 (2017).

[8] P. Marmottant, A. Mgharbel, J. Käfer, B. Audren, J.-P. Rieu, J. Vial, B. Van Der Sanden, A. Marée, F. Graner, and H. Delanoë-Ayari, Proc. Natl. Acad. Sci. U.S.A. 106, 17271 (2009).

[9] A. Mongera, P. Rowghanian, H. J. Gustafson, E. Shelton, D. A. Kealhofer, E. K. Carn, F. Serwane, A. A. Lucio, J. Giammona, and O. Campàs, Nature (London) 561, 401 (2018). 
[10] F. Serwane, A. Mongera, P. Rowghanian, D. A. Kealhofer, A. A. Lucio, Z. M. Hockenbery, and O. Campàs, Nat. Methods 14, 181 (2017).

[11] K. Guevorkian, M.-J. Colbert, M. Durth, S. Dufour, and F. Brochard-Wyart, Phys. Rev. Lett. 104, 218101 (2010).

[12] R. Vincent, E. Bazellières, C. Pérez-González, M. Uroz, X. Serra-Picamal, and X. Trepat, Phys. Rev. Lett. 115, 248103 (2015).

[13] A. R. Harris, L. Peter, J. Bellis, B. Baum, A. J. Kabla, and G. T. Charras, Proc. Natl. Acad. Sci. U.S.A. 109, 16449 (2012).

[14] S. Tlili, E. Gauquelin, B. Li, O. Cardoso, B. Ladoux, H. Delanoë-Ayari, and F. Graner, R. Soc. Open Sci. 5, 172421 (2018).

[15] M. Reffay, L. Petitjean, S. Coscoy, E. Grasland-Mongrain, F. Amblard, A. Buguin, and P. Silberzan, Biophys. J. 100, 2566 (2011).

[16] X. Serra-Picamal, V. Conte, R. Vincent, E. Anon, D. T. Tambe, E. Bazellières, J. P. Butler, J. J. Fredberg, and X. Trepat, Nat. Phys. 8, 628 (2012).

[17] K. Doxzen, S. R. K. Vedula, M. C. Leong, H. Hirata, N. S. Gov, A. J. Kabla, B. Ladoux, and C. T. Lim, Integr. Biol. 5, 1026 (2013).

[18] O. Cochet-Escartin, J. Ranft, P. Silberzan, and P. Marcq, Biophys. J. 106, 65 (2014).

[19] R. Farooqui and G. Fenteany, Cell 118, 51 (2005).

[20] T. Das, K. Safferling, S. Rausch, N. Grabe, H. Boehmand, and J. P. Spatz, Nat. Cell Biol. 17, 276 (2015).

[21] S. R. K. Vedula, M. C. Leong, T. L. Lai, P. Hersen, A. J. Kabla, C. T. Lim, and B. Ladoux, Proc. Natl. Acad. Sci. U.S.A. 109, 12974 (2012).

[22] G. Stokes, Trans. Camb. Phil. Soc. 9, 8 (1851).

[23] P. Saramito, Complex Fluids: Modelling and Algorithms (Springer, Heidelberg, 2016).

[24] I. Cantat, S. Cohen-Addad, F. Elias, F. Graner, R. Höhler, O. Pitois, F. Rouyer, and A. Saint-Jalmes, in Foams: Structure and Dynamics, edited by S. J. Cox (Oxford University Press, Oxford, 2013).

[25] D. L. Weaire and S. Hutzler, The Physics of Foams (Oxford University Press, Oxford, 1999).

[26] I. Cheddadi, P. Saramito, B. Dollet, C. Raufaste, and F. Graner, Eur. Phys. J. E 34, 1 (2011).

[27] E. Kolb, P. Cixous, N. Gaudouen, and T. Darnige, Phys. Rev. E 87, 032207 (2013).

[28] I. Zuriguel, D. R. Parisi, R. C. Hidalgo, C. Lozano, A. Janda, P. A. Gago, J. P. Peralta, L. M. Ferrer, L. A. Pugnaloni, E. Clément, D. Maza, I. Pagonabarraga, and A. Garcimartín, Sci. Rep. 4, 7324 (2015).
[29] J. H. Kim, X. Serra-Picamal, D. T. Tambe, E. H. Zhou, C. Y. Park, M. Sadati, J.-A. Park, R. Krishnan, B. Gweon, E. Millet, J. P. Butler, X. Trepat, and J. J. Fredberg, Nat. Mater. 12, 856 (2013).

[30] G. B. Blanchard, A. J. Kabla, N. L. Schultz, L. C. Butler, B. Sanson, N. Gorfinkiel, L. Mahadevan, and R. J. Adams, Nat. Methods 6, 458 (2009).

[31] I. Cheddadi, P. Saramito, and F. Graner, J. Rheol. 56, 213 (2012).

[32] See Supplemental Material at http://link.aps.org/ supplemental/10.1103/PhysRevLett.125.088102 for calculation of transport and rotation terms, movies, and additional figures.

[33] B. Lucas and T. Kanade, in Proceedings of the 7th International Joint Conference on Artificial Intelligence, Vancouver, 1981 (IJCAI'81) (Morgan Kaufmann, San Francisco, 1981), Vol. 2, pp. 674-679.

[34] M. Durande, S. Tlili, T. Homan, B. Guirao, F. Graner, and H. Delanoë-Ayari, Phys. Rev. E 99, 062401 (2019).

[35] B. Guirao, S. Rigaud, F. Bosveld, A. Bailles, J. López-Gay, S. Ishihara, K. Sugimura, F. Graner, and Y. Bellaïche, eLife 4, e08519 (2015).

[36] M. Merkel, R. Etournay, M. Popović, G. Salbreux, S. Eaton, and F. Jülicher, Phys. Rev. E 95, 032401 (2017).

[37] F. Graner, B. Dollet, C. Raufaste, and P. Marmottant, Eur. Phys. J. E 25, 349 (2008).

[38] S. Tlili, C. Gay, F. Graner, P. Marcq, F. Molino, and P. Saramito, Eur. Phys. J. E 38, 33 (2015).

[39] P. Lee and C. W. Wolgemuth, PLoS Comput. Biol. 7, e1002007 (2011).

[40] J. Étienne, J. Fouchard, D. Mitrossilis, N. Bufi, P. DurandSmet, and A. Asnacios, Proc. Natl. Acad. Sci. U.S.A. 112, 2740 (2015).

[41] H. Phillips and M. S. Steinberg, J. Cell Sci. 30, 1 (1978), https://jcs.biologists.org/content/30/1/1.article-info.

[42] J. Ranft, M. Basan, J. Elgeti, J.-F. Joanny, J. Prost, and F. Jülicher, Proc. Natl. Acad. Sci. U.S.A. 107, 20863 (2010).

[43] N. Jain, K. V. Iyer, A. Kumar, and G. V. Shivashankar, Proc. Natl. Acad. Sci. U.S.A. 110, 11349 (2013).

[44] G. Forgacs, R. A. Foty, Y. Shafrir, and M. S. Steinberg, Biophys. J. 74, 2227 (1998).

[45] E.-M. Schötz, R. Burdine, F. Jülicher, M. Steinberg, C.-P. Heisenberg, and R. Foty, HFSP J. 2, 42 (2008).

[46] M. Rauzi, in Cell Polarity and Morphogenesis, Methods in Cell Biology, Vol. 139 (Elsevier, Amsterdam, 2017), pp. 153-165.

[47] G. Erdemci-Tandogan, M. J. Clark, J. D. Amack, and M. L. Manning, Biophys. J. 115, 2259 (2018). 